\title{
Corela
}

Cognition, représentation, langage

HS-5 | 2006

Organisation des textes et cohérence des discours

\section{Cohésion-cohérence : accords et désaccords}

\section{Mathilde Salles}

\section{OpenEdition}

Journals

Édition électronique

URL : http://journals.openedition.org/corela/1426

DOI : $10.4000 /$ corela. 1426

ISSN : 1638-573X

\section{Éditeur}

Cercle linguistique du Centre et de l'Ouest - CerLICO

\section{Référence électronique}

Mathilde Salles, "Cohésion-cohérence : accords et désaccords », Corela [En ligne], HS-5 | 2006, mis en ligne le 27 octobre 2006, consulté le 19 avril 2019. URL : http://journals.openedition.org/corela/1426 ; DOI : 10.4000/corela.1426

Ce document a été généré automatiquement le 19 avril 2019.

\section{(c) (i) (2)(2)}

Corela - cognition, représentation, langage est mis à disposition selon les termes de la licence Creative Commons Attribution - Pas d'Utilisation Commerciale - Partage dans les Mêmes Conditions 4.0 International. 


\title{
Cohésion-cohérence : accords et désaccords
}

\author{
Mathilde Salles
}

\section{NOTE DE L'AUTEUR}

Je remercie F. Cornish pour ses précieuses remarques.

\section{Introduction}

1 La distinction entre les notions de cohésion et de cohérence est, comme le note M. Charolles (1988), plutôt bien établie depuis la fin des années $70^{1}$ :

« (...) tout le monde est à peu près d'accord pour opposer d'un côté la cohérence, qui a à voir avec l'interprétabilité des textes, et, de l'autre les marques de relation entre énoncés ou constituants d'énoncés. Concernant ces marques, depuis M.A.K. Halliday et R. Hasan (1976), on tend à les regrouper sous le nom générique de cohésion. » (M. Charolles 1988, 53)

2 Ce consensus se vérifie aisément dans les définitions proposées par les dictionnaires spécialisés : ceux-ci relient, en effet, régulièrement la cohérence à l'interprétabilité du texte ou du discours et la cohésion aux moyens linguistiques (anaphores, répétitions, ellipses, connecteurs ${ }^{2}$, etc.) qui permettent d'assurer lien et continuité ${ }^{3}$.

Lors d'une première approche du couple cohésion-cohérence, il est sans doute inévitable de se poser la plupart des questions qui ont alimenté et qui alimentent encore beaucoup de travaux sur le sujet. Des questions qui posent essentiellement le problème de leur solidarité et qui peuvent s'exprimer sous deux formes :

- la $1^{\text {ère }}$, extrêmement classique, pour ne pas dire rebattue, subordonne la cohérence à la cohésion et se demande ainsi dans quelle mesure la cohésion est un facteur de cohérence ; 
- la $2^{\text {nde }}$, sans doute moins classique, même si elle est loin d'être inédite, va, au contraire, subordonner la cohésion à la cohérence et se demander alors dans quelle mesure la cohérence est un facteur de cohésion.

4 C'est à la première question, beaucoup plus complexe qu'il n'y paraît, que je vais m'intéresser ici.

5 La cohésion est-elle un facteur de cohérence ? Et, plus précisément, en est-elle une condition nécessaire et suffisante ou seulement nécessaire mais non suffisante ou même ni nécessaire ni suffisante?

\section{Condition suffisante?}

6 Si la cohésion a souvent été présentée comme un facteur de cohérence, elle n'a jamais - à ma connaissance - été présentée comme un facteur exclusif et, par conséquent, comme une condition suffisante. Les travaux qui, notamment dans les années 70, se sont attachés à définir les conditions de cohérence ou, plus généralement les conditions de la création d'un texte, ont tous reconnu que la cohésion n'était pas le seul facteur de textualité. Par exemple, I. Bellert, en 1970, souligne déjà que la répétition, c'est-à-dire les reprises référentielles, ne constitue qu'une condition nécessaire mais non suffisante à la cohérence ${ }^{4}$. Cette condition de répétition représente, quelques années plus tard, l'une des quatre méta-règles de cohérence de Charolles (1978), les trois autres étant liées à la progression, la non-contradiction et la relation :

- «Méta-règle de répétition: Pour qu'un texte soit (microstructurellement ou macrostructurellement) cohérent, il faut qu'il comporte dans son développement linéaire des éléments à récurrence stricte. » (p.14)

- «Méta-règle de progression: Pour qu'un texte soit microstructurellement ou macrostructurellement cohérent, il faut que son développement s'accompagne d'un apport sémantique constamment renouvelé. » (p.20)

- «Méta-règle de non-contradiction: Pour qu'un texte soit microstructurellement ou macrostructurellement cohérent, il faut que son développement n'introduise aucun élément sémantique contredisant un contenu posé ou présupposé par une occurrence antérieure ou déductible de celle-ci par inférence. » (p.22)

- "Méta-règle de relation : Pour qu'une séquence ou un texte soient cohérents, il faut que les faits qu'ils dénotent dans le monde représenté soient directement reliés.»(p.32). Et Charolles évoque ici des relations comme la cause, la condition ou la conséquence. En fait, c'est sur ces relations que se fondent la plupart des recherches consacrées à la cohérence, des recherches qui étudient et définissent la cohérence à partir des relations sémantiques et pragmatiques, dites aussi relations de discours ou encore, depuis les travaux de J.R. Hobbs, relations de cohérence, telles que la Cause-Conséquence, l'Elaboration, le Contraste, l'Exemplification, la Généralisation, etc., qui s'établissent entre des segments de discours successifs.

7 On retrouve trois de ces quatre règles chez T. Reinhart (1980) sous les noms de 1. cohésion (comparable à la répétition de Bellert 1970 et Charolles 1978), 2. consistance (comparable à la non-contradiction) et 3. pertinence (qu'on rapprochera de la règle de relation de Charolles, même si l'extension de cette condition de pertinence semble plus large : elle inclut, en effet, outre les relations entre les phrases, les relations de celles-ci avec le thème du discours ou avec le contexte d'énonciation ; cf. Reinhart 1980, 164). 
Charolles affirme explicitement que ces conditions ne sont pas des conditions suffisantes. Et Reinhart l'illustre avec un exemple de texte cohésif mais non cohérent, parce qu'inconsistant (violation de sa deuxième condition) :

(1) I was living at home. But my father is dead now... That's why you can say he's probably decided to smoke a pipe [the interviewer was smoking a pipe]

(exemple extrait du discours enregistré d'un patient schizophrène, que Reinhart 1980, 164, reprend à Freeman, Cameron et McGhie 1966 ${ }^{5}$ )

La cohésion et la connexion entre la deuxième et la troisième phrase sont assurées par la reprise pronominale he et le connecteur that's why, mais, dans la mesure où ces deux phrases ne peuvent pas être vraies ensemble, dans la mesure où ce qu'elles décrivent est incompatible, l'ensemble paraît incohérent ${ }^{6}$. Avec un tel exemple, le caractère non suffisant de la cohésion pour la cohérence devient évident. Mais cette évidence semble avoir toujours besoin d'être réaffirmée, si l'on en juge par la liste constamment renouvelée d'exemples de textes cohésifs et apparemment incohérents. Des exemples souvent fabriqués de toutes pièces, plus rarement attestés (ces derniers étant relevés soit, comme celui de Reinhart, chez des patients schizophrènes, soit dans des copies de jeunes élèves ${ }^{7}$ ). La plupart de ces exemples présentent des coq-à-l'âne très cohésifs, autrement dit des séquences qui violent la condition de relation (Charolles) ou de pertinence (Reinhart). C'est le cas dans l'exemple (2), construit par J. Hobbs $(1979,67)$ et dans l'exemple (3), construit presque vingt ans plus tard par A. Reboul $(1997,199)$ :

(2) John took a train from Paris to Istanbul. He likes spinach.

(3) Jean a acheté une vache. Elle est rousse comme un écureuil. Il vit dans la forêt et hiberne l'hiver. Il est très froid dans la région.

On peut évoquer aussi des textes qui, bien que très cohésifs, sont incohérents par manque de progression. Il s'agit de textes extrêmement redondants, comme (4) et (5) proposés par Charolles $(1978,20-21$ et 21$)$; le premier, caricatural, est un texte qui se répète inlassablement, chaque phrase constituant une simple reformulation de la précédente :

(4) Les veuves ne reçoivent que la moitié de la retraite de leur feu mari. Les femmes non mariées perçoivent une pension égale à la moitié de celle que percevait leur mari défunt. Elles n'ont que cinquante pour cent des indemnités que touchait leur mari quand il était vivant. Du temps qu'il était en retraite les épouses des retraités partageaient avec leur mari la totalité de leur pension.

11 Le second, extrêmement répétitif, provient d'une copie d'élève :

(5) Le forgeron est vêtu d'un pantalon noir et d'un chapeau clair et d'une veste grise et marron foncé. Il tient à la main le piquet de la pioche et il tape dessus avec un marteau sur le piquet de la pioche. Les gestes qu'il a fait, il a le piquet de la pioche et avec son marteau il tape sur le piquet de la pioche. Le bout de cet outil qui s'appelle le piquet de la pioche est pointu et l'autre bout est carré. Pour le faire devenir rouge avec le piquet de la pioche, il l'a mis dans le feu et les mains sont rouges.

12 Ces textes ne pêchent pas par manque de cohésion; au contraire, c'est plutôt leur excès de cohésion qui, en interdisant toute progression, les rend confus, difficilement interprétables. La reformulation et la répétition ne constituent pas en elles-mêmes des facteurs d'incohérence; elles peuvent avoir des vertus pédagogiques et figurent d'ailleurs régulièrement dans l'inventaire des différentes relations de cohérence ${ }^{8}$. Autrement dit, elles représentent un des moyens de satisfaire l'une des conditions de cohérence posées par Charolles (1978) et Reinhart (1980) : la condition de relation ou de pertinence. Mais, et ce n'est peut-être pas une propriété qui leur est propre par rapport aux autres relations de cohérence, elles ne supportent pas l'excès. 


\section{Condition nécessaire?}

13 l'absence de consistance (i.e. la présence de contradictions) ou à l'absence de relation (ou pertinence). Ce qui représente des transgressions à trois des quatre règles de Charolles. Reste alors une dernière règle : la répétition ou plus généralement la cohésion. L'absence de cohésion est-elle un facteur d'incohérence comme peuvent l'être l'absence de progression, de consistance ou de relation? Je serais tentée d'apporter une réponse de Normande à cette question : oui et non.

On peut en fait distinguer deux types de manquements à la règle de cohésion, deux types aux conséquences très différentes :

- l'absence totale de marques cohésives constitue un premier manquement possible à cette règle,

- une mauvaise utilisation des marques cohésives en constitue un autre.

\subsection{La cohérence à l'épreuve d'une mauvaise utilisation des marques cohésives}

Une mauvaise utilisation des marques de cohésion peut sans conteste produire de l'incohérence. Evidemment, toute « erreur » ou maladresse dans le choix des expressions référentielles n'est pas source d'incohérence. Par exemple, la reprise par un article défini à la place d'un adjectif démonstratif dans un énoncé de reprise immédiate comme celui de G. Kleiber (1986a et b) :

(6) Un avion s'est écrasé hier à New York. Cet avion/? L'avion relie habituellement Miami à New York.

même si elle est maladroite, ne produit pas de problèmes d'interprétation insurmontables.

On opère malgré tout la coréférenciation entre l'avion et un avion. Les choses sont très différentes pour un exemple comme (7):

(7) Un étudiant $\mathrm{i}_{\mathrm{i}}$ est entré dans le bureau. Ce gros étudiant ${ }_{\mathrm{i}} /{ }^{*}$ Le gros étudiant $\mathrm{a}_{\mathrm{i}} \mathrm{a}$ demandé l'heure.

(M. Gross 1977, 118)

Cette fois, le choix du déterminant est essentiel pour l'interprétation du SN : coréférentiel à l'indéfini un étudiant si l'on emploie le démonstratif; en nécessaire disjonction référentielle avec ce premier SN si l'on emploie l'article défini. Donc l'utilisation du défini, combiné à la détermination nouvelle gros, sera source d'incohérence si le gros étudiant n'est pas autrement identifiable. Certaines transgressions majeures au fonctionnement ordinaire des chaînes de référence risquent ainsi de produire des multiplications référentielles indésirables ; c'est encore le cas dans l'exemple suivant :

(8) Un homme marche sur le trottoir ; soudain un homme glisse.

(copie d'un enfant déficient auditif; exemple transmis par C. Feuillard)

La condition de nouveauté attachée à l'emploi de l'indéfini interdira l'interprétation coréférentielle vraisemblablement désirée par le locuteur. Son énoncé risque donc d'être jugé incohérent, bien que cette condition de nouveauté ne soit qu'une condition par défaut (cf. notamment, F. Corblin 1994 et 1995) que le discours littéraire n'hésite pas à contourner9. 
18 Les expériences menées par A. Garnham, J. Oakhill et P.N. Johnson-Laird (1982) confirment cette importance de la maîtrise des expressions référentielles pour l'interprétation. Leurs expériences consistent à présenter à un groupe d'étudiants (expérience 1) ou à deux groupes d'enfants âgés de 7 à 8 ans ${ }^{10}$ (expérience 2) trois versions différentes de textes (histoires ou descriptions dans l'expérience 1 , histoires courtes dans l'expérience 2) - la version originale, une version reprenant les mêmes phrases que la version originale, mais présentées dans un ordre aléatoire, et une dernière version reprenant cet ordre aléatoire mais avec, cette fois, une révision des liens cohésifs. Voici un exemple de ces trois versions, emprunté à la deuxième expérience de Garnham, Oakhill et Johnson-Laird (1982, 41-42) :

(9) version originale

Jenny was holding on tightly to the string of her beautiful new balloon. She had just won it and was hurrying home to show her sister. Suddenly, the wind caught it and carried it into a tree. The balloon hit a branch and burst. Jenny cried and cried.

(10) version aléatoire

She had just won it and was hurrying home to show her sister. Suddenly, the wind caught it and carried it into a tree. Jenny was holding on tightly to the string of her beautiful new balloon. Jenny cried and cried. The balloon hit a branch and burst.

(11) version aléatoire modifiée

Jenny had just won a beautiful new balloon and was hurrying home to show her sister. Suddenly, the wind caught it and carried it into a tree. Jenny was holding on tightly to the string of her balloon. She cried and cried. It hit a branch and burst.

Dans la deuxième version - i.e. l'ordre aléatoire sans révision des liens cohésifs - on ne sait pas vraiment, comme le soulignent Garnham, Oakhill et Johnson-Laird, de quoi l'histoire parle et l'utilisation du pronom avant le nom propre ne permet plus d'opérer les coréférenciations nécessaires. Résultat, l'interprétation va multiplier les référents de discours: she et Jenny vont être traitées comme deux expressions en disjonction référentielle. Lorsque les liens cohésifs sont correctement utilisés, comme en (11), le problème tient alors seulement au défaut de plausibilité de la séquence («implausible sequence of events ", écrivent Garnham, Oakhill et Johnson-Laird, p. 42), ce qui relève finalement ici de la règle de relation et de la règle de non-contradiction, les deux étant liées dans cet exemple. C'est, en effet, l'incohérence de la séquence temporelle, l'incohérence dans l'ordre de déroulement des événements qui conduit à nous présenter Jenny tenant encore son ballon, alors que celui-ci s'est déjà envolé dans un arbre.

20 Et les expériences de Garnham, Oakhill et Johnson-Laird montrent que les versions « ordre aléatoire » sont moins bien mémorisées que les versions originales, mais aussi que la restitution de liens cohésifs corrects améliore généralement l'effet de compréhension et de mémorisation ${ }^{11}$.

\subsection{Absence de marques cohésives et cohérence : quelques exemples}

21 Jusqu'ici on a donc pu remarquer que, d'une part, la cohésion n'est nullement suffisante pour la cohérence, mais qu'elle peut aussi être cause d'incohérence lorsqu'elle est utilisée de manière excessive (cf. les exemples (4) et (5) empruntés à Charolles) ou lorsque ses marques sont mal maîtrisées comme dans les exemples (7) et (8). Reste à savoir si elle est nécessaire à la cohérence. En d'autres termes - et on retrouve là le premier manquement possible à la règle de cohésion que j'évoquais au début de cette section - un texte peut-il se dispenser de marques cohésives? Question plus délicate que la première. Pas de 
réponse unanime cette fois : pour certains, la cohésion n'est pas suffisante, mais elle est nécessaire (c'est le cas de Bellert 1970 et Halliday et Hasan 1976) ; pour d'autres, les plus nombreux, elle n'est ni suffisante ni nécessaire (c'est le cas, par exemple, de Brown et Yule 1983, Giora 1985, Charolles 1988, Moeschler et Reboul 1994, Cornish 1996, Fries 2004...).

Quelques exemples de textes non cohésifs, mais susceptibles de recevoir une lecture cohérente, devraient suffire à établir le caractère non nécessaire de la cohésion. Les exemples proposés par G. Brown et G. Yule $(1983,196)$ - exemples dans lesquels l'absence de marques cohésives n'empêche pas le lecteur de rétablir des relations sémantiques entre les phrases et de les interpréter comme un texte, comme le soulignent les auteurs sont tout à fait probants, seulement ils sont réduits à deux phrases. Les deux exemples choisis par J. Moeschler et A. Reboul (1994, 460), eux aussi réduits à deux phrases, me paraissent, en revanche, beaucoup moins probants. Ces deux exemples sont les suivants :

(12) Nous sommes allés au cinéma. La bière était fraîche.

(13) Nous aurons des invités à dîner. Calderon était un grand écrivain.

(exemple de Van Dijk cité par Charolles 1988, 51)

Ces deux exemples ont en commun, selon Moeschler et Reboul (1994, 461), l'absence de « relation référentielle ou anaphorique entre les énoncés qui les composent » et le fait d'être, malgré tout, interprétables. Le premier exemple est, en effet, facilement interprétable ${ }^{12}$, et cela sans aménagement trop compliqué (contrairement au second), à partir d'un schéma d'inférence comme celui que proposent Moeschler et Reboul :

(14) a. Si nous allons au cinéma, nous buvons une bière au bar pendant l'entracte.

b. Nous sommes allés au cinéma.

c. Nous avons bu une bière au bar.

Schéma d'inférence qui, en outre, explique pourquoi on peut employer la bière au défini ; je cite encore Moeschler et Reboul $(1994,461)$ :

«La présence d'un article défini en (25) [ = notre exemple (12)] s'explique par la relation associative existant entre cinéma et bière, via le schéma d'inférence donné en $\left(25^{\prime}\right)$ [ = (14) ici $》$

Finalement, la cohérence de la séquence - séquence censée être caractérisée par son absence de relation référentielle ou anaphorique - paraît crucialement liée à l'interprétation anaphorique associative du SN défini la bière par rapport à cinéma ou plutôt à aller au cinéma. Voilà qui est gênant pour un exemple destiné à illustrer l'indépendance de la cohésion et de la cohérence.

Le problème posé par l'exemple (13) est différent. Moeschler et Reboul reprennent à dessein un exemple de séquence jugée d'abord incohérente par son auteur, puis réhabilitée par les auteurs d'un compte-rendu de la thèse de Van Dijk, mais cela au prix de certaines acrobaties : pour conférer de la cohérence à l'ensemble, il faut imaginer une famille qui a l'habitude de rendre hommage chaque année à Calderon, en organisant un dîner en son honneur le jour de sa mort $^{13}$...

Ce que l'exemple choisi par Moeschler et Reboul souligne bien, c'est que la cohérence est une question d'interprétation et non une propriété intrinsèque des textes. Mais un tel exemple ne doit pas nous faire croire qu'une suite non cohésive n'acquiert de la cohérence qu'au prix de calculs et de reconstructions si complexes. Des exemples beaucoup plus simples et plus immédiatement cohérents comme (15) et (16) suffisent à montrer que la présence de marques cohésives n'est en rien nécessaire à l'interprétation cohérente d'une séquence de deux phrases : 
(15) Il a beaucoup plu. La rivière a débordé.

(G. Gross et M. Prandi 2004, 39)

(16) Albert siffla. Un lièvre détala.

(Charolles 1994, 138)

L'absence de marques de continuité n'entrave en rien une lecture cohérente de ces deux séquences, lesquelles reposent toutes les deux, et ce n'est pas un hasard, sur une inférence causale. Ce n'est pas un hasard car, en effet, les inférences causales semblent les plus immédiatement accessibles, les plus fortement cohérentes pourrait-on dire. C'est pourquoi la plupart des recherches consacrées à la cohérence commencent souvent par définir cette notion grâce à un enchaînement causal ou, plus exactement, grâce à un enchaînement qui conduit l'interprétant à une inférence causale. C'est le cas, par exemple, dans l'ouvrage d'A. Kehler $(2002,2)$ - ouvrage entièrement consacré à la cohérence, cohérence basée sur les relations, dans la lignée de Hobbs - qui, à la question Qu'est-ce que la cohérence? posée dans les premières pages de l'introduction, répond par l'exemple suivant :

(17) John took a train from Paris to Istanbul. He has family here. commenté en ces termes :

«Dans la plupart des situations de discours, on inférera probablement que le fait pour John d'avoir de la famille à Istanbul est la raison pour laquelle il prend un tel train. Bien que cette conclusion ne soit pas explicitement énoncée, elle est naturelle dès lors qu'on suppose que les énoncés entretiennent des relations les uns avec les autres, c'est-à-dire que le discours est cohérent. » (Kelher 2002, 2 ; je traduis et je souligne)

C'est-à-dire, comme Kehler le précisera juste après, qu'on peut inférer une relation causeeffet entre les propositions.

\subsection{Des relations de cohérence plus ou moins cohésives}

Cette prééminence ${ }^{14}$ des relations causales sur les autres relations de cohérence est régulièrement signalée dans les travaux consacrés à la cohérence ; par exemple :

- par C. Ruhl (1974), qui pose des degrés croissants de cohérence selon le lien interprété, le degré maximum étant représenté par la relation de cause ;

- par les travaux menés autour de T. Sanders (cf. notamment T. Sanders, W. Spooren et L. Noordman 1992, W. Spooren 1997, A. Knott et T. Sanders 1998 et T. Sanders et L. Noordman 2000)

- ou, plus récemment, par F. Cornish (2003 et à paraitre 2006), qui distingue des relations de cohérence à la force cohésive plus ou moins lâche.

31 Ces différences entre les relations de cohérence sont d'ailleurs confirmées par un certain nombre d'expériences psycholinguistiques: Knott et Sanders $(1998,138)$ évoquent à ce sujet des expériences qui montrent que les relations causales sont lues plus rapidement que les relations non causales ou encore que les événements reliés par des relations causales sont mieux mémorisés.

32 La distinction qu'opère Cornish (2003 et à paraître 2006) en termes de relations de cohérence à la force cohésive plus ou moins lâche me paraît particulièrement heureuse en ce qu'elle reflète, je crois, certains aspects du choix référentiel. Le choix des expressions référentielles et, par conséquent, le choix des marques cohésives semble parfois déterminé par le type de relation de cohérence entre les phrases et, plus précisément, par le degré de cohésion de ces relations de cohérence. 

relation de cohérence, c'est parce que les expressions référentielles elles-mêmes témoignent d'une force cohésive plus ou moins importante. Ces différences apparaissent nettement dans les travaux menés par G. Kleiber (1994), puis par C. Schnedecker (1997 et 2003), sur la distribution du pronom personnel et du nom propre dans des phrases consécutives. Ces travaux montrent, en effet, que le mode de saisie référentielle propre à ces deux types d'expressions a des incidences sur les relations sémantiques entre propositions contiguës : là où le pronom - qui, selon Kleiber (1994), saisit le référent en continuité avec ce qui l'a rendu saillant dans le contexte précédent - aura tendance à convoquer ou à renforcer un lien de causalité entre deux propositions, le nom propre qui, lui, toujours selon Kleiber $(1994,100)$, « ne peut que redonner le référent comme en première mention» - aura tendance à bloquer une telle relation et permettra, pour paraphraser Kleiber, d'exprimer d'autres contenus. D'autres contenus, autrement dit d'autres relations de cohérence et, plus précisément, des relations de cohérence à la force cohésive plus lâche, vraisemblablement des relations additives, des relations d'expansion comme la relation d'Elaboration ou la relation de Liste. Les exemples (18) et (19) empruntés à Schnedecker $(1997,85$ et 2003, 117) l'illustrent bien :

(18) Betty a soif. Betty boit.

(extrait d'un résumé du film Betty de Chabrol)

(19) Betty a soif. Elle boit.

Ces deux exemples permettent d'opposer pronom personnel et nom propre selon l' opération de base qui fonde la relation de cohérence, c'est-à-dire, dans les termes de Sanders, Spooren et Noordman (1992), selon le type causal (implicatif) ou simplement additif (non implicatif) de la relation de cohérence ${ }^{15}$. L'analyse de Schnedecker souligne en effet que la relation de causalité qui s'établit naturellement dans la version pronominale (i.e. Betty boit parce qu'elle a soif) est remplacée par une tout autre relation dans la version originale, avec répétition du nom propre: "le rapport de causalité disparaît, écrit Schnedecker $(2003,117)$, et (...), corollairement, on infère de l'enchaînement que Betty est alcoolique ${ }^{16}$. On pourrait ajouter que l'interprétation de la première phrase elle-même est affectée par cette répétition du nom propre dans la deuxième phrase, puisqu'elle perd, elle aussi, son interprétation ponctuelle pour une interprétation habituelle (i.e. Betty a toujours soif. Betty boit sans arrêt). Et, ainsi, la deuxième phrase apparaît comme une Elaboration, une forme de répétition de la première, puisque les deux nous permettent d'inférer la même proposition ${ }^{17}$ : « Betty est alcoolique ».

Cette corrélation entre lecture habituelle et répétition immédiate du nom propre s'observe assez fréquemment. Et ainsi, les différences d'interprétation provoquées par l'emploi du nom propre à la place du pronom se laissent souvent décrire non seulement en termes de relations de cohérence (par exemple, relation d'Elaboration ou de Liste versus relation de Cause/Conséquence), mais aussi en fonction d'une opposition entre lecture habituelle et lecture ponctuelle, entre propriété permanente et propriété épisodique du référent. J'emprunte encore une fois un exemple à Schnedecker $(1997,149)$ pour l'illustrer :

(20) Patrick en savait long sur le début de la carrière du docteur Lyte. Patrick était

dangereux.

(M. Spark, Les célibataires)

exemple qu'elle fait contraster avec la version pronominale (21) : 
(21) Patrick en savait long sur le début de la carrière du docteur Lyte. Il était dangereux. proches de ceux observés pour les exemples (18) et (19): la version pronominale s'accompagnera vraisemblablement d'une interprétation en termes de cause/ conséquence et ainsi le prédicat de la deuxième proposition (était dangereux) décrira une propriété épisodique, transitoire, du référent, alors que, dans la version originale, avec nom propre, ce même prédicat décrira une propriété stable, permanente, du référent, c'est-à-dire une propriété qui n'est pas uniquement la conséquence de la première proposition, mais qui, comme le souligne Schnedecker, vaut généralement pour le référent. La séquence s'interprétera probablement selon une structure Liste (cf. infra) i.e. « Patrick en savait long sur le début de la carrière du docteur Lyte. et, en plus de ça, Patrick était dangereux »- et non selon une structure Cause/Conséquence医 i.e. « Patrick en savait long sur le début de la carrière du docteur Lyte ; il était donc dangereux ».

\subsection{Absence de marques cohésives et cohérence : d'autres exemples?}

Mais revenons à nos séquences cohérentes sans marques cohésives. Les exemples, on l'a $\mathrm{vu}$, sont réduits à peu de phrases, en général deux. Et on peut se demander si, finalement, l'apparente divergence d'opinions sur le caractère nécessaire ou non de la cohésion n'est pas simplement liée aux différences de dimension des textes considérés. Si un texte très court peut se dispenser de marques cohésives, qu'en est-il d'un texte un peu développé ? Existe-t-il des textes non cohésifs vraiment développés? On a peut-être quelquefois un peu confondu une cohérence locale - cohérence entre des segments contigus qui, on l'a constaté avec des exemples comme (15) et (16), s'établit sans peine en l'absence de marques cohésives - avec une cohérence globale du texte, difficile à envisager s'il n'y a aucune marque de continuité topicale. Cette confusion des deux niveaux explique sûrement comment les mêmes auteurs, Moeschler et Reboul (1994), peuvent, après avoir souligné le caractère non nécessaire des relations de coréférence et d'anaphore pour la cohérence des discours (p.460), considérer les chaînes de référence et les chaînes anaphoriques comme un facteur important sinon indispensable de la cohérence ${ }^{18}$ des discours. La contradiction n'est qu'apparente et on peut légitimement considérer que si la cohésion n'est pas nécessaire à une cohérence locale, elle l'est sans doute à un niveau plus général, puisqu'elle constitue le principal moyen d'assurer la continuité topicale ${ }^{19}$. Or, outre l'existence de relations sémantiques ou pragmatiques entre les énoncés (règle de relation chez Charolles ou de pertinence chez Reinhart), la cohérence, et ici, en fait, la macro-cohérence, a aussi à voir, comme le rappelle fort justement Cornish $(1996,41)$, avec l'établissement et le maintien de domaines topicaux. Les marques cohésives ne suffisent évidemment pas à garantir une continuité topicale ; tous les exemples de coq-àl'âne fortement cohésifs, fréquemment utilisés pour affirmer le caractère non suffisant de la cohésion pour la cohérence, le soulignent. On se souvient ici même des exemples (2) et (3), construits respectivement par Hobbs (1979) et Reboul (1997), auxquels on peut ajouter l'exemple d'une lettre d'un patient schizophrène que Cornish $(1996,40-41)$ emprunte à Roberts et Kreuz $(1993,457)^{20}$ :

(22) I am writing on paper. The pen which I am using is from a factory called « Perry and Co.» This factory is in England. I assume this. Behind the name of Perry Co. the city of London is inscribed; but not the city. The city of London is in 
England. I know this from my school days. Then, I always like geography. My last teacher in the subject was August A. He was a man with black eyes. I also like black eyes. There are also blue and grey eyes and other sorts, too. I have heard it said that snakes have green eyes. All people have eyes. There are some, too, who are blind. These blind people are led about by a boy. It must be very terrible not to be able to see. There are people who can't see, and in addition, can't hear. I know some who hear too much. One can hear too much. There are many sick people in Burgholzli (sic); they are called patients.

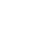

Impossible évidemment d'assurer une continuité topicale si aucun topique général n'est établi. L'incohérence d'un exemple comme celui fabriqué par Reboul (1997) - ici exemple (3) - relève à la fois d'une absence de relation entre les faits dénotés et d'une absence de topique global. Il est ainsi micro et macro-incohérent. Quant à la lettre donnée en (22), Cornish $(1996,41)$ remarque qu'elle pêche surtout par absence de topique global, un topique global qui permettrait de regrouper la multitude des topiques locaux ${ }^{21}$. Si la cohérence globale est fortement atteinte, la cohérence locale, en revanche, est parfois sauve.

Une fois un topique général établi, la continuité topicale pourra-t-elle être assurée indépendamment de la continuité référentielle et, ainsi, de la cohésion? On serait tenté de répondre par la négative. Mais ce serait sans doute aller vite en besogne et ignorer certains types de textes, des types de textes qui s'appuient sur des relations de cohérence faiblement cohésives. Garnham, Oakhill et Johnson-Laird (1982), lors de leur première expérience (cf. supra), portant sur des histoires et des descriptions, observent que les descriptions présentent peu de continuité référentielle d'une phrase à l'autre, chaque phrase ayant tendance à présenter un aspect différent du topique. La continuité des séquences descriptives est plus « diffuse » (p.37) que celle des histoires ${ }^{22}$. Cela dit, faible continuité référentielle, c'est-à-dire, finalement, rareté des liens de coréférence, ne signifie pas forcément faibles liens cohésifs et les descriptions sont souvent très fortement cohésives, même en l'absence de continuité référentielle au sens strict du terme. La description suivante, par exemple, présente un seul point de connexion (avec la combinaison mais surtout) et très peu de reprises anaphoriques coréférentielles (deux intraphrastiques - qui, son - et une seule transphrastique - $i l$, qui reprend le SN un fort bel établissement de la phrase précédente) :

(23) M. Lebigre tenait un fort bel établissement, d'un luxe tout moderne. Placé à l'encoignure droite de la rue Rambuteau, flanqué de quatre petits pins de Norvège dans des caisses peintes en vert, il faisait un digne pendant à la grande charcuterie des Quenu-Gradelle. Les glaces claires laissaient voir la salle, ornée de guirlandes de feuillages, de pampres et de grappes, sur un fond vert tendre. Le dallage était noir et blanc, à grands carreaux. Au fond, le trou béant de la cave s'ouvrait sous l'escalier tournant, à draperie rouge, qui menait au billard du premier étage. Mais le comptoir surtout, à droite, était très riche, avec son large reflet d'argent poli.

(Zola, Le ventre de Paris, Folio / Gallimard, 2002, p. 168)

Mais les liens cohésifs abondent entre les phrases et ce sont précisément sur ces liens-là que s'organise la séquence descriptive. Cette séquence énumère différents éléments de l'établissement de Lebigre en se fondant principalement sur des anaphores associatives ( les glaces claires, la salle, le dallage, au fond, la cave, l'escalier tournant, le premier étage, le comptoir). Ces anaphores associatives supposent à la fois un lien référentiel (surtout avec les premières phrases, mais aussi parfois entre phrases contiguës, comme c'est le cas pour la relation anaphorique qui s'établit entre la salle et le dallage) et un lien lexical puisque les 
anaphores se fondent soit sur une relation méronymique, soit sur la relation lexicale que Kleiber (2001) a décrite en termes de localisation fonctionnelle. Autrement dit, ces anaphores sont doublement cohésives. Chaque anaphorique introduit une nouvelle preuve venant confirmer les affirmations des deux premières phrases (concernant le luxe et la beauté de l'établissement de Lebigre, digne pendant de la charcuterie des QuenuGradelle) et toutes ces preuves s'organisent en série, selon une séquence Liste, au demeurant une des relations de cohérence les moins cohésives qui soient ${ }^{23}$. La relation de Liste est définie comme une relation additive (cf. Sanders, Spooren et Noordman 1992) qu'on peut " expliciter $»^{24}$ au moyen du connecteur et. Dans le cadre de la RST (Rhetorical Structure Theory) de W.C. Mann et S.A. Thompson, c'est une relation multinucléaire, sans limitation du nombre de noyaux (cf., par exemple, B. Fox 1987 ou W. Mann et M. Taboada 2005). Ces propriétés de la relation de Liste, c'est-à-dire le fait qu'elle soit additive et non implicative et qu'elle ne soit pas limitée en nombre de segments, en font une relation de cohérence privilégiée pour développer un texte cohérent mais non ou peu cohésif ${ }^{25}$. Dans la description de Zola le lien reste encore assez fort entre les différents éléments de la Liste, grâce notamment aux relations lexicales de co-méronymie ou de co-localisation fonctionnelle qui s'établissent entre la plupart des termes anaphoriques et grâce à la relation anaphorique qui unit la salle à le dallage. Mais la relation de Liste se dispense facilement de ce type de liens, comme l'illustre l'exemple (24), exemple choisi par Giora $(1985,18)$, puis par Cornish $(1996,40)$, pour souligner le caractère non nécessaire des liens cohésifs pour la cohérence :

(24) Every person constructs a world of his own, from his illusions and hopes, from his love and weakness. Kafka's Prague was only [the] Prague of his thoughts and eyes, Nahum Gutman's little Tel Aviv was created by his hands, Nathan Alterman invented a Jaffa of his own poem... Everyone lives in his own Israel, according to his powers and talents..

(extrait d'un journal hébreux traduit par Giora, corrigé par Cornish)

41 Comme le précise Cornish $(1996,40)$, on a ici un argument général confirmé par une série d'exemples (le Prague de Kafka, le Tel Aviv de Nahum Gutman et le Jaffa de Nathan Alterman), exemples distincts sans marques cohésives entre eux, qui forment une Liste. Donc une relation d'Exemplification (cf. Hobbs 1990, Kehler 2002, Cornish 2003), dans laquelle l'exemplification elle-même se développe selon une structure en Liste ; structure d'ensemble finalement assez proche de celle sur laquelle s'organise la description de Zola.

Selon Fox $(1987,81)$, dans la structure Liste, les membres sont présentés comme égaux. Cela dit, cette égalité est parfois modulée par des conjonctions et/ou adverbes dont le rôle est justement de hiérarchiser les éléments de la Liste, le plus souvent en la clôturant. C'est la fonction de mais et surtout à la fin de la description de Zola (« Mais le comptoir surtout, à droite, était très riche, avec son large reflet d'argent poli. »), c'est encore celle de l'adverbe even dans l'exemple (25), relevé par Fox $(1987,81)$ :

(25) He knew his Rousseau; he knew his Voltaire; he even knew his President de Brosses!

(Leon Edel, Bloomsbury : A House of Lions ; c'est moi qui souligne)

Mais lorsque l'égalité est parfaite, lorsque le texte n'utilise aucune de ces marques hiérarchiques et conclusives, l'ordre des éléments de la Liste paraît pratiquement interchangeable, et, cela, que ces éléments adoptent une progression à thème constant comme dans l'exemple (25) ou à thèmes dérivés comme dans les exemples (23) et (24). La relative indépendance et l'égalité de ces éléments entre eux explique qu'on ait là une des connexions les plus faibles qui soient (i.e. une des relations de cohérence les moins 
cohésives), elle explique vraisemblablement aussi qu'on puisse, en adoptant une progression à thèmes dérivés, développer assez longuement un texte indépendamment de toutes marques cohésives.

\section{Conclusion}

Finalement, expliquer l'absence de marques cohésives par la nature des relations de cohérence, c'est réaffirmer l'étroite solidarité entre la cohésion et la cohérence et c'est sûrement, aussi, laisser entrevoir le rôle primordial de la cohérence - et, plus précisément, des relations de cohérence - pour la cohésion, pour le choix des expressions référentielles et pour leur interprétation. Ce qui conduit naturellement à l'autre question fondamentale : la cohérence est-elle un facteur de cohésion?

\section{BIBLIOGRAPHIE}

Adam, J.-M. (2002), article « Cohérence » in P. Charaudeau et D. Maingueneau (2002), 99-100.

Bellert, I. (1970), « On a Condition of the Coherence of Texts », Semiotica, 4, 335-363.

Brown, G. et Yule, G . (1983), Discourse analysis, Cambridge, Cambridge University Press.

Charaudeau, P. et Maingueneau, D. (2002), Dictionnaire d'analyse du discours, Paris, Editions du Seuil.

Charolles, M. (1976), « Grammaire de texte - théorie du discours - narrativité », Pratiques, 11/12, 133-154.

Charolles, M. (1978), « Introduction aux problèmes de la cohérence des textes », Langue française, 38, 7-41.

Charolles, M. (1988), « Les études sur la cohérence et la connexité textuelles depuis la fin des années 1960 », Modèles linguistiques, tome X, fasc.2, n²0, 45-66.

Charolles, M. (1994), «Cohésion, cohérence et pertinence du discours », Travaux de linguistique, 29, 125-151.

Corblin, F. (1987), Indéfini, défini et démonstratif, Genève, Droz.

Corblin, F. (1994), « La condition de nouveauté comme défaut », Faits de langues, 4, 147-155.

Corblin, F. (1995), Les formes de reprise dans le discours, Rennes, Presses Universitaires de Rennes.

Cornish, F. (1996), « Coherence: the lifeblood of anaphora », Belgian Journal of Linguistics, vol. 10, 37-54.

Cornish, F. (2003), « Types de relations de discours entre énoncés : interactions avec l'anaphore transphrastique ", Unité(s) du texte, Cahier du CRISCO n 12, D. Legallois (éd.), Université de Caen, 69-84.

Cornish, F. (à paraître 2006), « Relations de cohérence et anaphores en contexte interphrastique : une symbiose parfaite », Langages, 164. 
Fox, B. (1987), Discourse structure and anaphora, Cambridge, Cambridge University Press.

Fries, P. H. (2004), « What makes a text coherent? », in Text and texture, D. Banks (éd.), Paris, L'Harmattan.

Garnham, A., Oakhill, J. et Johnson-Laird, P.N. (1982), « Referential continuity and the coherence of discourse ", Cognition, 11, 29-46.

Giora, R. (1985), « What's a coherent text? », in Text Connexity, Text Coherence: Aspects, Methods, Results, E. Sözer (éd.), Hambourg, Helmut Buske.

Gross, G. et Prandi, M. (2004), La finalité, Bruxelles, Duculot.

Gross, M. (1977), Grammaire transformationnelle du français. Syntaxe du nom, Paris, Larousse.

Halliday, M.A.K. et Hasan, R. (1976), Cohesion in English, Londres, Longman.

Hobbs, J.R. (1979), « Coherence and coreference », Cognitive Science, 3, 67-90.

Hobbs, J.R. (1990), Literature and Cognition, chapitre 5: « The Coherence and Structure of Discourse », Stanford University, CLSI Lecture Notes 21, 83-114.

Kehler, A. (2002), Coherence, Reference, and the Theory of Grammar, Stanford University, CSLI Publications.

Kleiber, G. (1986a), « Adjectif démonstratif et article défini en anaphore fidèle », in Déterminants : syntaxe et sémantique, J. David et G. Kleiber (éds), Paris, Klincksieck, 169-185.

Kleiber, G. (1986b), « Pour une explication du paradoxe de la reprise immédiate », Langue française , 72, 54-79.

Kleiber, G. (1994), Anaphores et pronoms, Louvain-la-Neuve, Duculot.

Kleiber, G. (2001), L'anaphore associative, Paris, PUF.

Knott, A. et Sanders, T. (1998), «The classification of coherence relations and their linguistic markers: An exploration of two languages », Journal of pragmatics, 30, 135-175.

Mann, W.C. et Thompson, S.A. (1988), « Rhetorical Structure Theory: Toward a functional theory of text organization », Text, 8 (3), 243-281.

Mann, W. et Taboada, M. (2005), RST Website, www.sfu.ca/rst.

Moeschler, J. et Reboul, A. (1994), Dictionnaire encyclopédique de pragmatique, Paris, Editions du Seuil.

Reboul, A. (1997), « (In)cohérence et anaphore : mythes et réalité », in Relations anaphoriques et (in)cohérence, W. De Mulder, L. Tasmowski-De Ryck et C. Vetters (éds), Amsterdam, Rodopi, 297-314.

Reinhart, T. (1980), « Conditions for text coherence », Poetics Today, vol. 1, n 4, 161-180.

Roberts, R.M. et Kreuz, R.J. (1993), « Nonstandard Discourse and Its Coherence », Discourse Processes, 16, 451-464.

Ruhl, C. (1974), « Semantic Anaphora », Papers in Linguistics, vol. 7, fasc.1-2, 205-238.

Salles, M. (2001), « Lorsqu'un SN indéfini n'introduit pas un référent nouveau : une affaire de point de vue ", L'information grammaticale, 91, 9-12.

Sanders, T. et Noordman, L. (2000), « The Role of Coherence Relations and Their Linguistic Markers in Text Processing », Discourse Processes, 29 (1), 37-60. 
Sanders, T., Spooren, W. et Noordman, L. (1992), « Toward a Taxonomy of Coherence », Discourse Processes, 15, 1-35.

Schnedecker, C. (1997), Nom propre et chaînes de référence, Paris, Klincksieck.

Schnedecker, C. (2003), « La question du nom propre répété dans la théorie dite du centrage et ses problèmes ", French Language Studies, 13, 105-134.

Spooren, W. (1997), « The Processing of Underspecified Coherence Relations », Discourse Processes, 24, 149-168.

Ushie, Y. (1986), « "Corepresentation" - A textual function of the indefinite expression », Text, 6, $4,427-446$.

\section{NOTES}

1. Fin des années 70 tout juste, puisque Charolles lui-même refusait de les distinguer en 1978, après avoir opposé en 1976 cohérence textuelle (en fait cohésion, selon l'acception classique) et cohérence discursive.

2. Les connecteurs sont parfois distingués des liens cohésifs comme marques de connexité.

3. Cf., par exemple, le chapitre 17 , intitulé «Cohérence: temporalité, relation thématique et enchaînement ", rédigé par J. Moeschler dans le Dictionnaire encyclopédique de pragmatique de J. Moeschler et A. Reboul (1994), et l'article «cohérence» de J.-M. Adam dans le Dictionnaire d'analyse du discours de P. Charaudeau et D. Maingueneau (2002).

4. Selon Halliday \& Hasan (1976, 324 ; je traduis) aussi, «dans la construction du texte, l'établissement de relations cohésives est un élément nécessaire ; mais ce n'est pas tout. ».

5. T. Freeman, J.L. Cameron et A. McGhie (1966), Studies in Psychosis, New York, International Universities Press.

6. On trouve une série d'exemples comparables chez Charolles (1978, 24-25), dans la section consacrée aux contradictions inférentielles et présuppositionnelles.

7. C'est le cas de plusieurs exemples donnés par M. Charolles (1978).

8. Cf., par exemple, la relation de Reformulation chez Mann et Thompson (1988) et Mann (1999), la relation Expository chez Halliday et Hasan (1976), ou encore certaines formes de la relation d' Elaboration chez Hobbs (1990) et Kehler (2002).

9. Voir Y. Ushie (1986), pour des exemples en anglais, F. Corblin (1987 et 1995) et M. Salles (2001), pour des exemples en français.

10. Deux groupes d'enfants distingués en fonction de leur capacité de compréhension (un groupe est dit « compétent » et l'autre « peu compétent »).

11. Sauf chez le groupe d'enfants "peu compétent », c'est-à-dire le groupe d'enfants ayant une capacité de compréhension moins élevée (cf. note précédente).

12. Même si l'exemple lui-même me paraît un peu difficile à accepter.

13. Cf. Charolles (1988) pour une présentation de la discussion autour de cet exemple et pour les références suivantes : O. Dahl et J. Dahl (1974), « Review of T.A. Van Dijk, Some aspects of text grammars ", Probleme und Perspectiven der neueren textgrammatischen Forschung, Papiere zur Textlinguistik 5, Hambourg, Buske, le compte-rendu de T.A. Van Dijk (1972), Some aspects of textgrammars, La Haye, Mouton.

14. Que David Hume, nous rappelle Charolles (1994, 139), avait déjà observée dans ses Essais philosophiques sur l'entendement humain (1748).

15. L'opération de base, qui constitue l'un des quatre primitifs qui fondent la classification des relations de cohérence proposée par Sanders, Spooren et Noordman (1992) - les trois autres étant la source de la cohérence (sémantique ou pragmatique), l'ordre des segments (marqué ou non) et 
la polarité de la relation (positive ou négative) - semble être un paramètre décisif pour le choix des expressions référentielles.

16. Avec cette fois l'interprétation - « être alcoolique » - la plus fréquemment associée à l'emploi absolu du verbe boire.

17. Cf. à ce sujet, la définition de l'Elaboration donnée par Hobbs $(1990,95)$ : «Inférer la même proposition $\mathrm{P}$ à partir des assertions de $\mathrm{S}_{0}$ et $\mathrm{S}_{1}$ ", définition que $\mathrm{F}$. Cornish propose ici même de compléter, afin qu'elle ne se confonde pas avec une relation de stricte paraphrase, de la manière suivante: il faut que $S_{1}$ «fournisse à la proposition inférée commune des informations supplémentaires, et que $\mathrm{e}_{1} \subseteq \mathrm{e}_{0}$ (l'événement évoqué par $\mathrm{S}_{1}$ doit faire partie de celui désigné par $\left.\mathrm{S}_{0}\right) »$.

18. Moeschler et Reboul $(1994,465)$ écrivent en fait qu'il est « difficile d'envisager un discours sans reprise anaphorique (chaîne anaphorique et chaîne référentielle) ».

19. A ce propos, on pourrait rapprocher cohésion et progression : si, localement, la progression n'est pas nécessaire (les reformulations sont possibles), elle semble, en revanche, indispensable à une cohérence globale. Cf. supra, en particulier les exemples (4) et (5), empruntés à Charolles (1978).

20. Lettre qu'ils reprennent eux-mêmes à E. Bleuler (1950), Dementia praecox or the group of schizophrenias, New York, International Universities Press.

21. Cornish (1996) fait en outre remarquer, à la suite de Roberts et Kreuz (1993), que la multiplicité des liens cohésifs contribue même à rendre l'ensemble encore plus incohérent, phénomène que nous avons aussi noté pour les exemples qui, tels (4) et (5), pêchent par absence de progression.

22. Et les descriptions sont ainsi «moins sensibles à la présentation en ordre aléatoire » (Garnham, Oakhill et Johnson-Laird 1982, 46).

23. Cf., entre autres, Spooren (1997) ou encore Sanders et Noordman $(2000,43)$ : « list relations are generally regarded as one of the weakest connections between two segments ».

24. " Expliciter ", le mot est fort étant donné le caractère peu explicite de cette conjonction.

25. On a déjà noté (cf. supra) le lien privilégié entre ce type de relation de cohérence et les répétitions immédiates de noms propres, expressions référentielles à la force cohésive plus lâche que les pronoms.

\section{RÉSUMÉS}

La cohésion est-elle un facteur de cohérence? En est-elle une condition nécessaire et suffisante ou seulement nécessaire mais non suffisante ou même ni nécessaire ni suffisante ? Ces questions, extrêmement classiques, seront l'occasion de montrer combien le choix des expressions référentielles et, par conséquent, le choix des marques cohésives peut être déterminé par le type de relation de cohérence (ou relation de discours) entre énoncés et, plus précisément, par le degré de cohésion de ces relations de cohérence. Notre étude, en soulignant cette étroite solidarité entre la cohésion et la cohérence, nous conduira naturellement à une autre question fondamentale : la cohérence est-elle un facteur de cohésion?

Is cohesion a necessary and sufficient condition for coherence? Or is it only a necessary one? Or is it neither necessary nor sufficient? Answering these very classic questions, I will emphasize the effect that coherence relations (or discourse relations) 澄 which are themselves more or less 
cohesive 50 have on referential choice, and so on the choice of cohesive ties. Our first questions will then be replaced by another significant one: is coherence a factor of cohesion?

INDEX

Mots-clés : cohésion, cohérence, anaphore, choix référentiel, relations de cohérence

Keywords : anaphora, referential choice, coherence relations

\section{AUTEUR}

MATHILDE SALLES

Université de Caen, CRISCO (CNRS - FRE 2805) 